\title{
LEFT ANNIHILATOR OF IDENTITIES WITH GENERALIZED DERIVATIONS IN PRIME AND SEMIPRIME RINGS
}

\author{
MD HAMIDUR RAHAMAN \\ Department of Mathematics \\ Aligarh Muslim University, Aligarh, 202002, India \\ e-mail: rahamanhamidmath@gmail.com
}

\begin{abstract}
Let $R$ be a noncommutative prime ring of char $(R) \neq 2, F$ a generalized derivation of $R$ associated to the derivation $d$ of $R$ and $I$ a nonzero ideal of $R$. Let $S \subseteq R$. The left annihilator of $S$ in $R$ is denoted by $l_{R}(S)$ and defined by $l_{R}(S)=\{x \in R \mid x S=0\}$. In the present paper, we study the left annihilator of the sets $\left\{F(x) \circ_{n} F(y)-x \circ_{n} y \mid x, y \in I\right\}$ and $\left\{F(x) \circ_{n} F(y)-d\left(x \circ_{n} y\right) \mid x, y\right.$ $\in I\}$.
\end{abstract}

Keywords: prime ring, derivation, generalized derivation, extended centroid, Utumi quotient ring.

2010 Mathematics Subject Classification: 16W25, 16R50, 16 N60.

\section{REFERENCES}

[1] S. Ali and H. Shuliang, On derivations in semiprime rings, Algebra Represent Theor. 15 (2012) 1023-1033.

doi:10.1007/s10468-011-9271-9

[2] M. Ashraf, A. Ali and R. Rani, On generalized derivations of prime rings, Southeast Asian Bull. Math. 29 (2005) 669-675.

[3] M. Ashraf and N. Rehman, On commutativity of rings with derivations, Results Math. 42 (2002) 3-8.

doi:10.1007/BF03323547

[4] H.E. Bell and N. Rehman, Generalized derivations with commutativity and anticommutativity conditions, Math. J. Okayama Univ. 49 (2007) 139-147.

[5] H.E. Bell and W.S. Martindale III, Centralizing mappings of semiprime rings, Canad. Math. Bull. 30 (1987) 92-101.

doi:10.4153/CMB-1987-014-x 
[6] K.I. Beidar, Rings of quotients of semiprime rings, Vestnik Moskow. Univ. Ser. I. Mat. Mekh. (Engl. Transl: Moskow Univ. Math. Bull.) 33 (1978) 36-42.

[7] C.L. Chuang, Hypercentral derivations, J. Algebra 166 (1994) 34-71. doi:10.1006/jabr.1994.1140

[8] C.L. Chuang, GPIs having coefficients in Utumi quotient rings, Proc. Amer. Math. Soc. 103 (1988) 723-728. doi:10.1090/S0002-9939-1988-0947646-4

[9] B. Dhara, S. Ali and A. Pattanayak, Identities with generalized derivations in semiprime rings, Demonstratio Math. XLVI (2013) 453-460. doi:10.1515/dema-2013-0471

[10] T.S. Erickson, W.S. Martindale III and J.M. Osborn, Prime nonassociative algebras, Pacific J. Math. 60 (1975) 49-63. doi:10.2140/pjm.1975.60.49

[11] I.N. Herstein, Topics in Ring Theorey (The University of Chicago Press, Chicago, 1969).

[12] S. Huang, Generalized derivations of prime rings, Int. J. Math. \& Math. Sci. Volume 2007, Article ID 85612, 6 pages. doi: $10.1155 / 2007 / 85612$

[13] N. Jacobson, Structure of rings, Amer. Math. Soc. Colloq. Pub. 37, Amer. Math. Soc. (Providence, RI, 1964).

[14] V.K. Kharchenko, Differential identity of prime rings, Algebra and Logic. 17 (1978) $155-168$. doi:10.1007/BF01669313

[15] T.K. Lee, Generalized derivations of left faithful rings, Comm. Algebra 27 (1999) 4057-4073. doi:10.1080/00927879908826682

[16] T.K. Lee, Semiprime rings with differential identities, Bull. Inst. Math. Acad. Sinica 20 (1992) 27-38.

[17] W.S. Martindale III, Prime rings satisfying a generalized polynomial identity, J. Algebra 12 (1969) 576-584. doi:10.1016/0021-8693(69)90029-5

[18] M.A. Raza and N.U. Rehman, On generalized derivation in rings and Banach Algebras, Kragujevac J. Math. 41 (2017) 105-120. doi:10.5937/KgJMath1701105R

Received 31 January 2020

Revised 16 May 2020

Accepted 16 May 2020 